\title{
Treatment Planning CT Radiomics for Predicting Treatment outcomes and Hematologic Toxicities to Intensity-modulated Radiation Therapy in Locally Advanced Cervical Cancer
}

\author{
Kang Ren ${ }^{1}$, Lin Shen ${ }^{1}$, Jianfeng Qiu ${ }^{2}$, Kui Sun ${ }^{2}$, Tingyin Chen ${ }^{1}$, Long Xuan ${ }^{3}$, Minwu \\ Yang $^{4}$, Hao-Yuan She ${ }^{5}$, Liangfang Shen ${ }^{1}$, Lan Deng ${ }^{6}$, Di Jing ${ }^{1}$, and Liting Shi ${ }^{2}$ \\ ${ }^{1}$ Xiangya Hospital Central South University \\ ${ }^{2}$ Shandong First Medical University \\ ${ }^{3}$ Central South University Xiangya School of Medicine \\ ${ }^{4}$ Central South University Xiangya School of Stomatology \\ ${ }^{5}$ Central South University School of Life Sciences \\ ${ }^{6}$ Hunan University of Technology
}

March 2, 2022

\begin{abstract}
Objectives: We evaluated radiotherapy planning CT-based radiomics for predicting clinical endpoints [tumor complete response (CR), 5-year overall survival (OS), hypohemoglobin, and leucopenia] after intensity-modulated radiation therapy (IMRT) in locally advanced cervical cancer (LACC). Methods: This study retrospectively collected 257 LACC patients treated with IMRT from 2014 to 2017. Patients were allocated into the training/validation sets (3:1 ratio) using proportional random sampling, resulting in the same proportion of groups in the two sets. We extracted 254 radiomic features from each of the gross target volume (GTV), pelvis, and sacral vertebrae in planning CT images. The sequentially backward elimination support vector machine algorithm was used for feature selection and endpoint prediction. Model performance was evaluated using area under the curve (AUC). Results: A combination of 10 clinicopathological parameters and 34 radiomic features achieved the best performance for predicting CR [validation balanced accuracy: 80.79\%]. For OS, 54 radiomic features showed good prediction accuracy [validation balanced accuracy: $85.75 \%$ ], and the threshold value of their scores can stratify patients into the low-risk and high-risk groups $(\mathrm{P}<0.001)$. The clinical and radiomic models were also predictive of hypohemoglobin and severe leucopenia [validation balanced accuracies: $70.96 \%$ and $69.93 \%$ ]. Conclusion: This study demonstrated that combining clinicopathological parameters with CT-based radiomics had good predictive value for treatment outcomes and hematologic toxicities to radiotherapy in LACC. The prediction of clinical endpoints prior to radiotherapy may assist the radiation therapists to select the optimal therapeutic strategy with the minimal toxicity and best curative effect.
\end{abstract}

Title: Treatment Planning CT Radiomics for Predicting Treatment Outcomes and Hematologic Toxicities to Intensity-modulated Radiation Therapy in Locally Advanced Cervical Cancer

Kang Ren ${ }^{1,2 \#, ~ L i n ~ S h e n ~}{ }^{1 \#}$, Jianfeng Qiu ${ }^{3}$, Kui Sun ${ }^{3}$, Tingyin Chen ${ }^{4}$, Xuan Long ${ }^{5}$, Minwu Yang ${ }^{6}$, Hao-Yuan She $^{7}$, Liangfang Shen ${ }^{1}$, Lan Deng ${ }^{8}$, Di Jing ${ }^{1 *}$, Liting Shi ${ }^{9 *}$

1. Department of Oncology, National Clinical Research Center for Geriatric Disorders, Xiangya Hospital, Central South University, Changsha 410008, China.

2. Department of Radiation Oncology, Peking Union Medical College Hospital, Chinese Academy of Medical Sciences \& Peking Union Medical College, Beijing 100730, China. 
3. Medical Science and Technology Innovation Center, Shandong First Medical University \& Shandong Academy of Medical Sciences, Jinan 250000, China.

4. Department of Network and Information Center, Xiangya Hospital, Central South University, Changsha 410008, China.

5. XiangYa School of Life Medicine, Central South University, Changsha 410013, China.

6. Xiangya School of Stomatology, Central South University, Changsha 410013, China.

7. School of Life Science, Central South University, Changsha 410013, China.

8. Hunan Polytechnic of Environment and Biology, Hengyang 421000, China.

9. Department of Radiology, Shandong First Medical University \& Shandong Academy of Medical Sciences, Taian 271016, China.

\#K.R. and L.S. contributed equally to this work.

*Co-corresponding authors

Liting Shi, Department of Radiology, Shandong First Medical University \& Shandong Academy of Medical Sciences, Changcheng Road No. 619, Taian 271016, Shandong, China. Tele: 86-0538-6222138, Email:

ltshi@foxmail.com

Di Jing, Department of Oncology, Xiangya Hospital, Central South University, Xiangya Road No. 87, Changsha 410008, Hunan, China. Tele: 86-0731-89753733, Email: 14092382@qq.com

Title: Treatment Planning CT Radiomics for Predicting Treatment Outcomes and Hematologic Toxicities to Intensity-modulated Radiation Therapy in Locally Advanced Cervical Cancer

\section{Abstract}

Objectives : We evaluated radiotherapy planning CT-based radiomics for predicting clinical endpoints [tumor complete response (CR), 5-year overall survival (OS), hypohemoglobin, and leucopenia] after intensitymodulated radiation therapy (IMRT) in locally advanced cervical cancer (LACC).

Design : A retrospectively study was performed between 2014 and 2017.

Setting : Xiangya hospital of Central South University, Changsha, Hunan, China.

Population : A total of 257 LACC patients were treated with IMRT.

Methods : Patients were allocated into the training/validation sets (3:1 ratio) using proportional random sampling, resulting in the same proportion of groups in the two sets.

Main outcomes and measures : The primary outcomes were the treatment response and hematologic toxicities caused by radiotherapy. We extracted 254 radiomic features from each of the gross target volume (GTV), pelvis, and sacral vertebrae in planning CT images. The sequentially backward elimination support vector machine algorithm was used for feature selection and endpoint prediction. Model performance was evaluated using area under the curve (AUC).

Results : A combination of 10 clinicopathological parameters and 34 radiomic features achieved the best performance for predicting CR [validation balanced accuracy: 80.79\%]. For OS, 54 radiomic features showed good prediction accuracy [validation balanced accuracy: 85.75\%], and the threshold value of their scores can stratify patients into the low-risk and high-risk groups $(\mathrm{P}<0.001)$. The clinical and radiomic models were also predictive of hypohemoglobin and severe leucopenia [validation balanced accuracies: $70.96 \%$ and $69.93 \%]$.

Conclusion : This study demonstrated that combining clinicopathological parameters with CT-based radiomics had good predictive value for treatment outcomes and hematologic toxicities to radiotherapy in 
LACC. The prediction of clinical endpoints prior to radiotherapy may assist the radiation therapists to select the optimal therapeutic strategy with the minimal toxicity and best curative effect.

Funding :

Keywords : Radiation Oncology; Treatment Planning CT, Radiomics; Treatment Outcome; Locally Advanced Cervical Cancer

\section{Abbreviations}

AUC: area under the curve

BM: bone marrow

CCRT: concurrent radiotherapy and chemotherapy

CI: confidence interval

CR: complete response

CT: computed tomography

EBRT: external beam radiotherapy

FDR: false discovery rate

FIGO: International Federation of Gynecology and Obstetrics

GLCM: gray level cooccurrence matrix

GLRLM: gray-level run length matrix

GOH: gradient orient histogram

GTV: gross target volume

ICC: intra-class coefficient

IMRT: intensity-modulated radiation therapy

LACC: locally advanced cervical cancer

LVSI: lymph-vascular space invasion

MRI: magnetic resonance images

NCCN: National Comprehensive Cancer Network

NIDM: neighborhood intensity difference matrix OS: overall survival

PD: progressive disease

PET: positron emission tomography

PR: partial response

RECIST: Response Evaluation Criteria In Solid Tumors

ROC: receiver operating characteristic

ROI: region of interest

SD: stable disease

\section{Introduction}


Cervical cancer is the fourth most commonly diagnosed cancer and the fourth leading cause of mortality among women worldwide. There were over 570,000 new cases annually and over 311,000 deaths in 2018 globally [1]. More than two-thirds of the patients are diagnosed with locally advanced cervical cancer (LACC) [2]. Concurrent chemoradiotherapy (CCRT) can lead to good prognosis for LACC [3], and the brachytherapy as a part of standard treatment regimen has further improved local control and overall survival, but the chemoradiotherapy resistance and local relapse also occur among $40 \%$ of LACC patients $[4,5]$. Early treatment response correlates to tumor response and survival [6]. Hematologic toxicity is the most common acute side effect of CCRT, which affect treatment efficacy and sometimes can be life-threatening [7]. Pretreatment prediction of early tumor response, survival, and hematologic toxicities may assist physicians to adjust radiotherapy planning and further improve the quality of life.

Previous studies demonstrated that the predictive efficacy of clinicopathological model for treatment outcomes is limited in LACC $[8,9]$. The therapeutic effect can be markedly different even in the same clinical stage. Currently, radiomics, a technique that can extract high-throughput quantitative features from imaging such as CT, PET, and MRI, has emerged as a promising tool for the assessment of treatment outcomes $[10,11]$. Radiomics can reflect the tumor intrinsic properties and can be used as independent predictors of survival outcome with higher predictive ability than traditional clinical parameters alone [12, 13].

Radiomics has been found to be related to lymph node metastases [14, 15], treatment response [16-18], patient survival [19], and tumor recurrence [16, 20] for cervical cancer patients [21]. So far as we know, there are no studies to investigate the radiomics predictive ability of $\mathrm{CT}$ images from radiotherapy planning system. Additionally, myelosuppression is the most common toxicity in pelvic radiotherapy with the Grade 3 or higher hematological toxicities of more than 30\% [22]. Bone marrow is the most radiosensitive pelvic organ, and approximately $40 \%$ of the total body BM reserve lies within the pelvic bones, especially pelvis and sacral vertebrae, which always are within the irradiated fields [23]. The individual differences of the radiationinduced hematologic toxicities caused even by the similar treatment regimens. Previously, radiomics was used to quantify lumbar and femoral mineral loss [24], mineral bone density, and osteoblast activity [25]. Treatment planning CT-based radiomic features of pelvis and sacral vertebrae may be able to predict the hematologic toxicities.

Therefore, we retrospectively examined radiotherapy planning CT images to establish radiomic models for predicting tumor complete response (CR), 5-year overall survival (OS) as well as hematologic toxicities (leucopenia and hypohemoglobin) in LACC patients. We also compared the prediction performance of clinicopathological parameters and radiomic features to further construct a combined model, aiming at better prediction of clinical endpoints in LACC.

\section{Materials and methods}

\subsection{Patients andimage datasets}

This retrospective study enrolled a cohort of 257 LACC patients treated with intensity-modulated radiotherapy (IMRT) in Xiangya Hospital between 2014 and 2017 (Table 1). Patient inclusion criteria were as follows: (a) patients had histologically proven carcinoma of the uterine cervix, staged IB2-IVA [26]; (b) patients were treated with pelvic external beam radiotherapy (EBRT) and subsequent brachytherapy with or without concurrent chemotherapy; (c) patients who received repeated pelvic MRI 3 months after the end of radiotherapy. Exclusion criteria were as follows: (a) patients who underwent neoadjuvant chemotherapy or radical surgery before radiotherapy; (b) patients with a diagnosis of other cancers; (c) patients with no available planning CT images.

The planning CT images were acquired using a Siemens CT scanner (SOMATOM Definition AS) with scanning voltages, tube currents, and exposure time of 100-140 kVp, 39-473 mA and 500-1000 ms, respectively. The pixel sizes of the CT images were $0.7 \mathrm{~mm} \times 0.7 \mathrm{~mm}$ to $1 \mathrm{~mm} \times 1 \mathrm{~mm}$; the slice thicknesses range from $3 \mathrm{~mm}$ to $5 \mathrm{~mm}$.

We also collected 88 of 257 cases with available fat-suppressed contrast-enhanced MRI T1-weighted images 
to compare the performance of CT and MRI radiomic features. Details are in Supplementary Materials.

\subsection{Treatment regimen}

All patients underwent EBRT followed by high-dose-rate intravaginal irradiation. EBRT was administered with a daily fraction of 1.8 Gy, 5 days a week, 25-28 times with a total dose of 45-50.4 Gy. For patients with positive para-aortic lymph nodes, extended-field radiotherapy that covered both the pelvis and the para-aortic nodal region was delivered. High-dose-rate intravaginal irradiation was delivered with a fraction of 6 Gy (twice per week) for a total dose of 30-36 Gy after EBRT.

The concurrent chemotherapy consisted of two main regimens: 1) Weekly single-agent cisplatin regimen repeated at 7-day intervals for four to six cycles; 2) Triweekly combination regimen, repeated at 21-day intervals for 1-3 cycles. Adjuvant chemotherapy regimen consisted of the combination of docetaxel $\left(75 \mathrm{mg} / \mathrm{m}^{2}\right)$ and cisplatin $\left(75 \mathrm{mg} / \mathrm{m}^{2}\right)$, repeated at 21-day intervals after completion of CCRT. Patients with liver/kidney dysfunction and other conditions were administrated with radiotherapy alone. The decision on the administration of adjuvant chemotherapy was based on the status of tumor regression.

\subsection{Imaging segmentation}

We defined three regions of interest (ROIs) in CT images, including gross target volume (GTV), pelvis, and sacral vertebrae (Figure 1). GTV consisting of the visible primary tumor and positive pelvic lymph node was previously defined for radiotherapy planning based on both CT and MRI (contrast-enhanced T1weighted images, T2-weighted images, DWI images) by the consensus of experienced radiation oncologists and radiologists. The size and morphology of all visible lymph nodes were inspected on CT or MRI images by two oncologists (10 years of experience) and reviewed by one radiologist (30 years of experience). A positive lymph node was a rounded soft-tissue structure with a short-axis diameter $>10 \mathrm{~mm}$ or with central necrosis $[27,28]$. The pelvis and sacral vertebrae were contoured independently by two radiologists (10 years of experience) and confirmed by a radiologist (30 years of experience). We used radiomic features of GTV to predict treatment outcomes, while those of pelvis and sacral vertebrae to predict hematologic toxicities. In addition, one radiologist ( 7 years of experience) independently contoured GTVs for 30 of 257 CT cases to test the inter-rater reliability of radiomic features.

\subsection{Assessment of treatment outcomes and hematologic toxicities}

The short-term tumor response was assessed based on pelvic MRI examination 3 months after radiotherapy according to the Response Evaluation Criteria In Solid Tumors v. 1.1 [29]: (1) CR represents the disappearance of all cervical lesions; (2) partial response (PR) represents at least a $30 \%$ decrease in the longest tumor diameter; (3) progressive disease (PD) represents at least a [?] 20\% increase in the longest diameter of tumor; and (4) stable disease (SD) represents neither sufficient decrease to qualify for PR nor sufficient increase in longest diameter for PD. OS was defined as the time from the date of diagnosis until death or the last follow-up. Hematologic toxicities during chemoradiation were assessed according to the National Cancer Institute Common Terminology Criteria for Adverse Events CTCAE 3.0 [29].

We investigated four clinical endpoints. (1) we categorized patients into the CR group and the non-CR group to predict tumor regression. (2) patients were divided into two groups by the cutoff OS time of 5 years. Binary classification models were used to predict whether patients survived [?] 5 years. (3) we predicted whether patients suffered from severe leucopenia (grade [?] 3) and (4) hypohemoglobin (grade $>0$ ) that could reflect treatment tolerance and influence outcome. Table 2 shows the number of patients stratified by the clinical endpoints.

Additionally, we used one-class learning algorithm to identify patients at high risk of treatment failures (SD and PD) due to the small sample size (5 SD and 4 PD of 257 patients). Methods and results are in supplementary materials.

\subsection{Feature extraction}


We extracted radiomic features using an open-source software IBEX (Figure 1) [30], including shape features, first-order features, and texture features. Shape features describe the tumor volume, surface area, and etc. First-order features were statistical descriptors of the image intensity, intensity histogram, and gradient orient histogram. Texture features included features calculated based on the neighborhood intensity difference matrix, gray-level cooccurrence matrix, and gray-level run length matrix. Texture features in different 3D directions were averaged as the final feature values. All of the images were rescaled into 100 gray levels before extracting texture features to avoid the generation of sparse matrices. The images were rescaled into 256 gray levels before extracting first-order features. No filter was applied to the images.

\subsection{Feature selection and endpoint prediction}

All patients were allocated into the training/validation sets (3:1 ratio) using proportional random sampling, in order to avoid unbalanced data distribution in the two sets. All clinicopathological data and radiomic features were normalized using robust data scaling method, which ignores the outliers when calculating the mean and standard deviation and then scales the variables using the calculated values. A four-step method was used to select predictive features and to build prediction models. First, the radiomic features with the inter-rater reliability of intra-class coefficient (ICC) $>0.80$ were selected. Second, the Lilliefors test was used to test whether data come from a normal distribution. We calculated the differences between two groups of patients using a two-sample two-sided t-test or Wilcoxon rank sum test depending on the normality of data. The difference in pelvic lymph node status was calculated using chi-square test. The radiomic features with $\mathrm{P}<0.05$ were selected. If the number of the selected features was $<20$ based on this criterion, $\mathrm{P}<$ 0.1 was used instead. The selected radiomic features and all clinicopathological parameters were candidate features for next step. Third, we classified two groups of patients and selected the best predictors using sequential backward elimination-support vector machine (SBE-SVM) algorithms. This method initially used all features to train and test an SVM model with a linear kernel in a five-fold cross validation using data in the training set and sequentially removed one feature from the feature set to see whether the prediction accuracy was improved or remained the same. If so, this feature was permanently removed. The soft margin SVM algorithm that is not sensitive to outliers was used for modelling, in order to prevent overfitting. The SBE-SVM model considers each feature's contribution to the classification task and finally gives the optimal combination of features, and has shown good performance in previous studies [31-33]. Finally, the final SVM model was used to predict classes of patients in the validation set. Please note that the performance of the training set was evaluated in a five-fold cross validation.

A receiver operating characteristic (ROC) curve was plotted using actual labels and the scores predicted by models as well as an area under curve (AUC) was simultaneously calculated as the major metric to evaluate the model performance. Besides, we also calculated accuracy, sensitivity, specificity, and F1-score as auxiliary metrics (definitions are in supplementary materials).

We established three models for prediction: a clinical model built using only clinicopathological parameters, a radiomic model built using only radiomic features, and a clinical and radiomic model built using both of them.

All analyses were performed using MATLAB 2018a. The SBE-SVM algorithm is based on MATLAB functions: 'sequentialfs' and 'fitcsvm'. The computational codes are available upon request to the corresponding author.

\section{Results}

\subsection{Feature extraction and selection}

We extracted 254 radiomic features from each ROI in CT images and collected 11 clinicopathological parameters for analysis. Table 1 shows the differences in 11 clinicopathological parameters between two groups of patients stratified by four clinical endpoints. The selected radiomic features and/or clinicopathological parameters in each model for four clinical endpoints are in Supplementary Table S1.

\subsection{Prediction of CR}


A combination of 10 clinicopathological parameters and $34 \mathrm{CT}$ radiomic features performed best in predicting CR [balanced accuracies: $79.73 \%$ and $80.79 \%$; AUC (95\% confidence interval (CI)): 0.88 (0.82-0.93) and 0.87 (0.77-0.96) in the training and validation sets]. The CT radiomic model outperformed clinicopathological model in predicting CR (Table 3). Figure 2a shows the ROC curves of three models for classifying the CR and non-CR groups. Additionally, only using the tumor volume to predict CR achieved balanced accuracies of $64.92 \%$ and $72.87 \%$ in the training and validation sets.

The CT radiomic model outperformed MRI radiomic model for predicting CR (balanced accuracy: $74.51 \%$ and $52.46 \%$ in the validation set), and the addition of MRI radiomic features decreased the performance of the clinical and radiomic model (Table S2 and Figure S1).

\subsection{Prediction of OS}

The radiomic model performed best in predicting OS [balanced accuracies: $78.69 \%$ and $85.75 \%$; AUC $(95 \%$ CI): $0.82(0.69-0.94)$ and $0.92(0.82-1)$ in training and validation sets]. Additionally, the balanced accuracies of only using the tumor volume to predict OS were $50 \%$ and $50 \%$ in the training and validation sets.

The scores predicted by the models were used to split patients into the high-risk and low-risk groups using the threshold value defined by the ROC curve of the training set. The radiomic scores can significantly stratify patients into the high-risk and low-risk groups $\left(\mathrm{P}=7.16 \times 10^{-10}\right)$, while clinical score cannot $(\mathrm{P}=$ 0.29) (Figure 3) [34].

The clinical model only showed limited predictive power for OS, and combining clinicopathological parameters with radiomic features didn't improve the performance (Table 3 and Figure $2 b$ ).

\subsection{Prediction ofhypohemoglobin}

We identified 9 clinicopathological parameters and 7 pelvis radiomic features as best predictors of hypohemoglobin [balanced accuracies: $62.42 \%$ and $70.96 \%$; AUC (95\% CI): 0.65 (0.57-0.73) and 0.74 (0.62-0.87) in training and validation sets]. The clinical model outperformed radiomic model in predicting hypohemoglobin (Table 3 and Figure 2c).

\section{4 Prediction of severeleucopenia}

The clinical model and the radiomic model alone only showed limited predictive power for severe leucopenia [balanced accuracies: $56.42 \%$ and 55.08\%; AUC (95\% CI): 0.56 (0.41-0.71) and 0.57 (0.43-0.72) in the validation set]. Combining radiomic features with clinicopathological parameters improved the prediction performance [balanced accuracy: 69.93\%; AUC (95\% CI): 0.64 (0.48-0.79) in validation set] (Table 3 and Figure 2d).

Figure 4 shows boxplots and data distribution of the predicted scores for four clinical endpoints.

We repeated the whole process using data of 159 patients treated with the same therapy. Similar performance was observed in the prediction of four clinical endpoints (Table S3 and Figure S2-S4).

The wide and overlapping 95\% CIs cannot represent statistical differences between two models.

\section{Discussion}

Radiation resistance is an independent poor prognostic factor, and adverse reactions affect tumor response and long-term survival for LACC. This study integrated clinicopathological parameters and treatment planning CT-based radiomics for predicting CR, 5-year OS, and hematologic toxicities. The clinical and radiomic models outperformed the single models (validation balanced accuracies: $80.79 \%$ vs $70.34 \%$ and $75.24 \%$ ) in predicting CR, indicating that a hybrid approach may have greater power for CR. For predicting OS, the radiomic model showed superior performance (validation balanced accuracy: 85.75\%). Although only using the tumor volume to build SVM models can predict short-term CR with good performance (validation balanced accuracy: $72.87 \%$ ), it showed low predictive power for long-term OS (validation balanced accuracy: $50 \%$ ). To predict hematologic toxicities, combining radiomic features with clinicopathological parameters can 
achieve validation balanced accuracies of $69.93 \%$ and $70.96 \%$ for severe leucopenia and hypohemoglobin, respectively. Treatment planning CT radiomics of bone marrow may be potential biomarkers for the prediction of treatment outcomes and hematologic toxicities in LACC.

Previous studies have demonstrated that tumor volume, pelvic lymph node status, and concurrent chemotherapy are independent prognostic factors of LACC [35]. But Lucia et al. reported that radiomic models have higher prognostic power than clinicopathological parameters, such as FIGO stage, tumor volume, and nodal stage [36]. Fang et al. constructed a multihabitat MRI radiomic model to predict tumor response with an AUC of 0.8 [37]. Our models integrated the $\mathrm{CT}$ radiomic features with clinicopathological parameters to predict $\mathrm{CR}$ achieved an AUC of 0.87, which outperformed the clinical (AUC 0.75) model, CT radiomic model (AUC 0.85), and MRI model (AUC 0.80). For predicting 5-year OS, our radiomic model reached an AUC of 0.82 , the clinical and radiomic model only achieved an AUC of 0.81 . The predictive power of the joint model is reduced possibly because different treatment regimens were performed to 257 patients. After the models were re-trained using 159 patients treated equally, the clinical and radiomic model achieved better performance than the radiomic model (Table S3). Previously, Ho et al. and Lucia utilized MRI radiomics to predict disease-free survival for cervical cancer patients, but the absence of long-term survival led the both studies to be limited $[36,38]$. We predicted both short-term CR and long-term survival, which help radiation therapists distinguish radio-resistance candidates in the early stage and adjusted treatment regimen in time such as the addition of radio-sensitizers and/or more intensive follow-up.

Bone marrow cells are easily damaged by low-dose radiation, which may be associated with hematologic toxicities. Previous studies have found the correlation between hematologic toxicities and dose-volume parameters of pelvic bone marrow based on PET-CT in rectal cancer and gynecological oncology [39, 40]. Utilizing CT radiomics of the pelvis and sacral vertebrae to predict hematologic toxicities has not yet been reported. We combined clinicopathological parameters and CT radiomics to predict hypohemoglobin (grade $>0$ ) and severe leucopenia (grade [?] 3) with AUCs of 0.74 and 0.64. The decreased hemoglobin levels are associated with the prognosis of radiotherapy and hypoxia-induced radio-resistance [41, 42]. Severe leucopenia increases the risk of infection and radiotherapy often needs to be suspended when it happens, which negatively affects the therapeutic efficacy. The accurate and timely prediction of hematologic toxicities may help to avoid serious complications. Future studies will take dosimetric factors of pelvis and sacral vertebrae into account to further improve the predictive power of our models for hematologic toxicities.

There are some limitations in our study. (i) Our results were not externally validated. External and multicentric data are needed to validate our results. (ii) The radiomic predictors for hematologic toxicities were extracted from CT images, which cannot fully present bone function alterations and bone-related diseases. Other imaging techniques, such as bone mineral densitometry, PET, and multiparametric MRI, may provide more predictive information for analyzing radiation-induced hematologic toxicities. (iii) We only studied planning CT and contrast-enhanced MRI T1-weighted images for predicting treatment outcomes without considering other CT/MRI sequences and modalities that may reveal underlying information for prediction. Further research is needed to investigate multimodality radiomic models and refine the optimal combination of clinical and imaging radiomic features.

In conclusion, noninvasive models based on clinicopathological parameters and treatment planning CT radiomics had predictive power for CR, 5-year OS, and hematologic toxicities. Before clinical application, external validation with a larger cohort is needed to refine the models for predicting the risk of treatment failure and hematologic toxicities.

\section{Conflicts of interests}

The authors declare no potential conflicts of interest.

\section{Author contributions}

Di Jing and Liting Shi conceived of the presented idea and supervised the project. Kang Ren, Lin Shen, Minwu Yang, Xuan Long, Tingyin Chen, and Hao-Yuan She prepared the sample and collected the data. Kui 
Sun, Kang Ren and Di Jing defined the regions of interest and assessed treatment response and Hematologic Toxicities. Liangfang Shen examined treatment response. Lin Shen, Lan Deng, and Liting Shi organized the data and calculated the radiomics features. Liting Shi and Jianfeng Qiu designed the computational model and analyzed the data. Kang Ren, Lin Shen, Di Jing and Liting Shi wrote the manuscript. All authors provided critical feedback and helped shape the analysis and manuscript.

\section{Ethics approval}

Ethical approval was granted by The ethics committee of Xiangya Hospital granted ethical approval on November 27, 2020. The informed consent obtained from all patients.

\section{Funding Statements}

This study was supported by the Academic Promotion Programme of Shandong First Medical University (2019QL009), the Taishan Scholars Program of Shandong Province (No. ts201712065), and National Natural Science Foundation of China (82060429).

\section{Acknowledgment}

Thanks to the Network and Information Center of Xiangya Hospital, which has given support in data extraction and analysis. Ethics was approved by the ethics committee of Xiangya Hospital and with informed consent obtained from all patients.

\section{References}

1. Arbyn, M., E. Weiderpass, L. Bruni, et al., Estimates of incidence and mortality of cervical cancer in 2018: a worldwide analysis. The Lancet. Global health, 2020. 8 (2): p. e191-e203.

2. Marquina, G., A. Manzano, and A. Casado, Targeted Agents in Cervical Cancer: Beyond Bevacizumab. Current oncology reports, 2018.20 (5): p. 40.

3. Cohen, P.A., A. Jhingran, A. Oaknin, and L. Denny, Cervical cancer. Lancet, 2019. 393 (10167): p. 169-182.

4. Quinn, M.A., J.L. Benedet, F. Odicino, et al., Carcinoma of the Cervix Uteri. International journal of gynaecology and obstetrics: the official organ of the International Federation of Gynaecology and Obstetrics, 2006. 95 Suppl 1.

5. Potter, R., K. Tanderup, M.P. Schmid, et al., MRI-guided adaptive brachytherapy in locally advanced cervical cancer (EMBRACE-I): a multicentre prospective cohort study. The Lancet. Oncology, 2021.22 (4): p. $538-547$.

6. Kong, S.-Y., K. Huang, C. Zeng, X. Ma, and S. Wang, The association between short-term response and long-term survival for cervical cancer patients undergoing neoadjuvant chemotherapy: a system review and meta-analysis. Scientific reports, 2018. 8 (1): p. 1545.

7. Kirwan, J.M., P. Symonds, J.A. Green, et al., A systematic review of acute and late toxicity of concomitant chemoradiation for cervical cancer. Radiotherapy and oncology : journal of the European Society for Therapeutic Radiology and Oncology, 2003. 68 (3): p. 217-226.

8. Gadducci, A., M.E. Guerrieri, and S. Cosio, Adenocarcinoma of the uterine cervix: Pathologic features, treatment options, clinical outcome and prognostic variables. Crit Rev Oncol Hematol, 2019.135 : p. 103114.

9. Rose, P.G., J. Java, C.W. Whitney, et al., Nomograms Predicting Progression-Free Survival, Overall Survival, and Pelvic Recurrence in Locally Advanced Cervical Cancer Developed From an Analysis of Identifiable Prognostic Factors in Patients From NRG Oncology/Gynecologic Oncology Group Randomized Trials of Chemoradiotherapy. Journal of clinical oncology : official journal of the American Society of Clinical Oncology, 2015. 33 (19): p. 2136-2142. 
10. Park, H., K.A. Kim, J.-H. Jung, J. Rhie, and S.Y. Choi, MRI features and texture analysis for the early prediction of therapeutic response to neoadjuvant chemoradiotherapy and tumor recurrence of locally advanced rectal cancer. European radiology, 2020.30 (8): p. 4201-4211.

11. Zhenyu, Liu, Shuo, et al., The Applications of Radiomics in Precision Diagnosis and Treatment of Oncology: Opportunities and Challenges. Theranostics, 2019.

12. Yip, S.S.F. and H.J.W.L. Aerts, Applications and limitations of radiomics. Physics in medicine and biology, 2016. 61 (13): p. R150-R166.

13. Verma, V., C.B. Simone, S. Krishnan, et al., The Rise of Radiomics and Implications for Oncologic Management. Journal of the National Cancer Institute, 2017. 109 (7).

14. Wu, Q., S. Wang, X. Chen, et al., Radiomics analysis of magnetic resonance imaging improves diagnostic performance of lymph node metastasis in patients with cervical cancer. Radiother Oncol, 2019.138 : p. 141148.

15. Wang, T., T. Gao, J. Yang, et al., Preoperative prediction of pelvic lymph nodes metastasis in earlystage cervical cancer using radiomics nomogram developed based on T2-weighted MRI and diffusion-weighted imaging. Eur J Radiol, 2019. 114 : p. 128-135.

16. Gao, S., S. Du, Z. Lu, et al., Multiparametric PET/MR (PET and MR-IVIM) for the evaluation of early treatment response and prediction of tumor recurrence in patients with locally advanced cervical cancer.Eur Radiol, 2020. 30 (2): p. 1191-1201.

17. Fang, M., Y. Kan, D. Dong, et al., Multi-Habitat Based Radiomics for the Prediction of Treatment Response to Concurrent Chemotherapy and Radiation Therapy in Locally Advanced Cervical Cancer.Front Oncol, 2020. 10 : p. 563.

18. Sun, C., X. Tian, Z. Liu, et al., Radiomic analysis for pretreatment prediction of response to neoadjuvant chemotherapy in locally advanced cervical cancer: A multicentre study. EBioMedicine, 2019. 46 : p. 160-169.

19. Fang, J., B. Zhang, S. Wang, et al., Association of MRI-derived radiomic biomarker with disease-free survival in patients with early-stage cervical cancer. Theranostics, 2020. 10 (5): p. 2284-2292.

20. Lucia, F., D. Visvikis, M. Vallieres, et al., External validation of a combined PET and MRI radiomics model for prediction of recurrence in cervical cancer patients treated with chemoradiotherapy. Eur J Nucl Med Mol Imaging, 2019. 46 (4): p. 864-877.

21. Tian, X., C. Sun, Z. Liu, et al., Prediction of Response to Preoperative Neoadjuvant Chemotherapy in Locally Advanced Cervical Cancer Using Multicenter CT-Based Radiomic Analysis. Front Oncol, 2020.10 : p. 77 .

22. Wang, W., X. Hou, J. Yan, et al., Outcome and toxicity of radical radiotherapy or concurrent Chemoradiotherapy for elderly cervical cancer women. BMC cancer, 2017. 17 (1): p. 510.

23. Mell, L.K., J.D. Kochanski, J.C. Roeske, et al., Dosimetric predictors of acute hematologic toxicity in cervical cancer patients treated with concurrent cisplatin and intensity-modulated pelvic radiotherapy. International journal of radiation oncology, biology, physics, 2006. 66 (5): p. 1356-1365.

24. Rastegar, S., M. Vaziri, Y. Qasempour, et al., Radiomics for classification of bone mineral loss: A machine learning study. Diagn Interv Imaging, 2020. 101 (9): p. 599-610.

25. Budan, F., K. Szigeti, M. Weszl, et al., Novel radiomics evaluation of bone formation utilizing multimodal (SPECT/X-ray CT) in vivo imaging. PLoS One, 2018. 13 (9): p. e0204423.

26. Pecorelli, S., L. Zigliani, and F. Odicino, Revised FIGO staging for carcinoma of the cervix. International Journal of Gynecology \& Obstetrics, 2009. 105 (2): p. 107-108. 
27. Choi, S.H., S.H. Kim, H.J. Choi, B.K. Park, and H.J. Lee,Preoperative magnetic resonance imaging staging of uterine cervical carcinoma: results of prospective study. Journal of computer assisted tomography, 2004. 28 (5): p. $620-627$.

28. Choi, H.J., S.H. Kim, S.-S. Seo, et al., MRI for pretreatment lymph node staging in uterine cervical cancer. AJR. American journal of roentgenology, 2006. 187 (5): p. W538-W543.

29. Eisenhauer, E.A., P. Therasse, J. Bogaerts, et al., New response evaluation criteria in solid tumours: revised RECIST guideline (version 1.1). Eur J Cancer, 2009. 45 (2): p. 228-47.

30. Zhang, L., D.V. Fried, X.J. Fave, et al., IBEX: an open infrastructure software platform to facilitate collaborative work in radiomics. Medical physics, 2015. 42 (3): p. 1341-1353.

31. Koutsouleris, N., L. Kambeitz-Ilankovic, S. Ruhrmann, et al.,Prediction Models of Functional Outcomes for Individuals in the Clinical High-Risk State for Psychosis or With Recent-Onset Depression: A Multimodal, Multisite Machine Learning Analysis. JAMA Psychiatry, 2018. 75 (11): p. 1156-1172.

32. Saeys, Y., I. Inza, and P. Larranaga, A review of feature selection techniques in bioinformatics. Bioinformatics, 2007.23 (19): p. 2507-2517.

33. Jiang, W., Y. Song, Z. Sun, J. Qiu, and L. Shi, Dosimetric Factors and Radiomics Features Within Different Regions of Interest in Planning CT Images for Improving the Prediction of Radiation Pneumonitis. Int J Radiat Oncol Biol Phys, 2021. 110 (4): p. 1161-1170.

34. Jordan H. Creed, T.A.G., Anders E. Berglund, MatSurv: Survival analysis and visualization in MAT$L A B$. Journal of Open Source Software, 2020. 5 (46): p. 1830.

35. Cohen, P.A., A. Jhingran, A. Oaknin, and L. Denny, Cervical cancer. Lancet (London, England), 2019. 393 (10167): p. 169-182.

36. Lucia, F., D. Visvikis, M.-C. Desseroit, et al., Prediction of outcome using pretreatment F-FDG PET/CT and MRI radiomics in locally advanced cervical cancer treated with chemoradiotherapy. European journal of nuclear medicine and molecular imaging, 2018. 45 (5): p. 768-786.

37. Fang, M., Y. Kan, D. Dong, et al., Multi-Habitat Based Radiomics for the Prediction of Treatment Response to Concurrent Chemotherapy and Radiation Therapy in Locally Advanced Cervical Cancer.Frontiers in oncology, 2020. $10:$ p. 563.

38. Ho, J.C., P.K. Allen, P.R. Bhosale, et al., Diffusion-Weighted Magnetic Resonance Imaging as a Predictor of Outcome in Cervical Cancer After Chemoradiation. International journal of radiation oncology, biology, physics, 2017. 97 (3): p. 546-553.

39. Kuncman, Ł., K. Stawiski, M. Masłowski, J. Kucharz, and J. Fijuth,Dose-volume parameters of MRIbased active bone marrow predict hematologic toxicity of chemoradiotherapy for rectal cancer.Strahlentherapie und Onkologie : Organ der Deutschen Rontgengesellschaft ... [et al], 2020. 196 (11).

40. Rose, B.S., Y. Liang, S.K. Lau, et al., Correlation between radiation dose to ${ }^{1} 8 F-F D G-P E T$ defined active bone marrow subregions and acute hematologic toxicity in cervical cancer patients treated with chemoradiotherapy. International journal of radiation oncology, biology, physics, 2012. 83 (4): p. 1185-1191.

41. Grigiene, R., K.P. Valuckas, E. Aleknavicius, J. Kurtinaitis, and S.R. Letautiene,

The value of prognostic factors for uterine cervical cancer patients treated with irradiation alone. BMC cancer, 2007. 7: p. 234.

42. Bishop, A.J., P.K. Allen, A.H. Klopp, L.A. Meyer, and P.J. Eifel,Relationship between low hemoglobin levels and outcomes after treatment with radiation or chemoradiation in patients with cervical cancer: has the impact of anemia been overstated? International journal of radiation oncology, biology, physics, 2015. 91 (1): p. 196-205. 


\section{Table and Figure legends}

Table 1. Patient characteristics $(\mathrm{n}=257)$.

Table 2. Clinical endpoints of patients $(\mathrm{n}=257)$.

Table 3. The prediction accuracy of treatment outcomes and hematologic toxicities.

Figure 1 . Schematic of data collection and analysis in the study.

Figure 2. The receiver operating characteristic (ROC) curves for discriminating between the two groups of patients divided by tumor complete response (a), 5-year overall survival (b), hypohemoglobin (c), and leucopenia (d) in the training and validation sets. The performance of the training set was evaluated in a five-fold cross validation.

Figure 3. The Kaplan-Meier Curves of patients in the high-risk and low-risk groups stratified using the threshold value of the predicted scores in the clinical model (a), the radiomic model (b), and the clinical and radiomic model (c). The threshold values were defined by the ROC curve of the training set. Patients censored $<5$ years were merged with the validation test when plotting the Kaplan-Meier Curves.

Figure 4. Boxplots and data distribution of the scores of all patients predicted by the clinical model (a), the radiomic model (b), and the clinical and radiomic model (c) for four clinical endpoints.
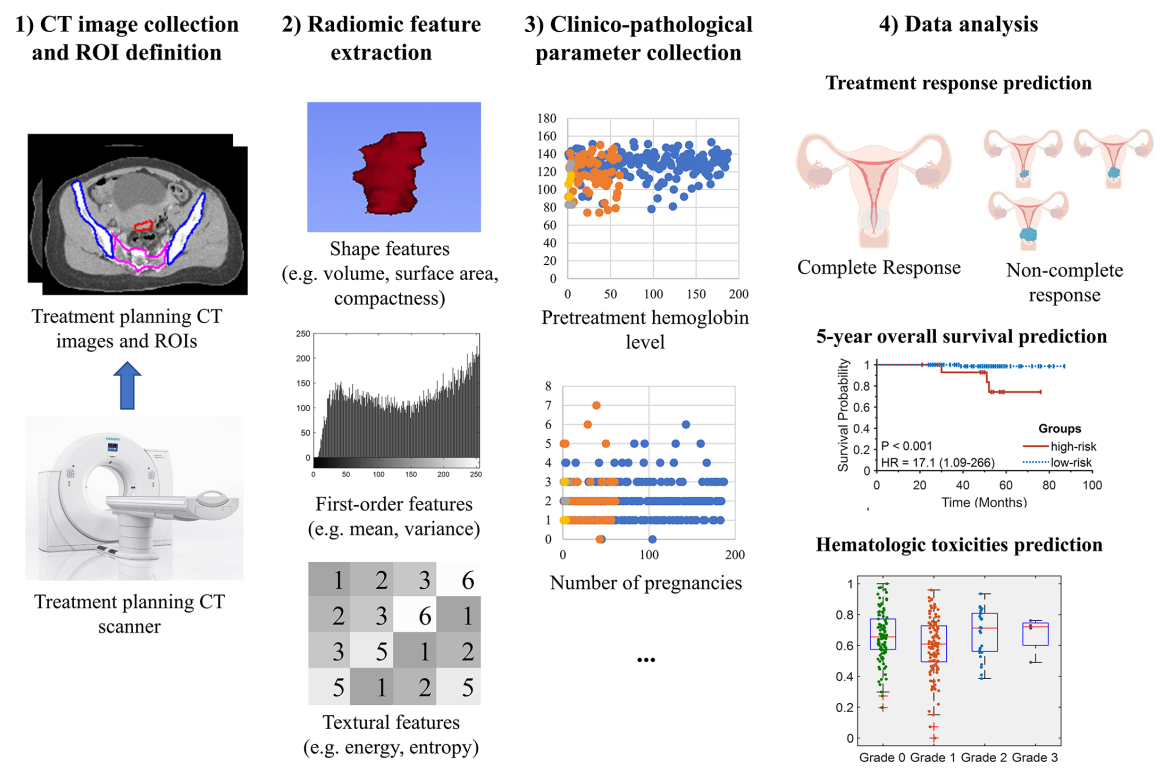

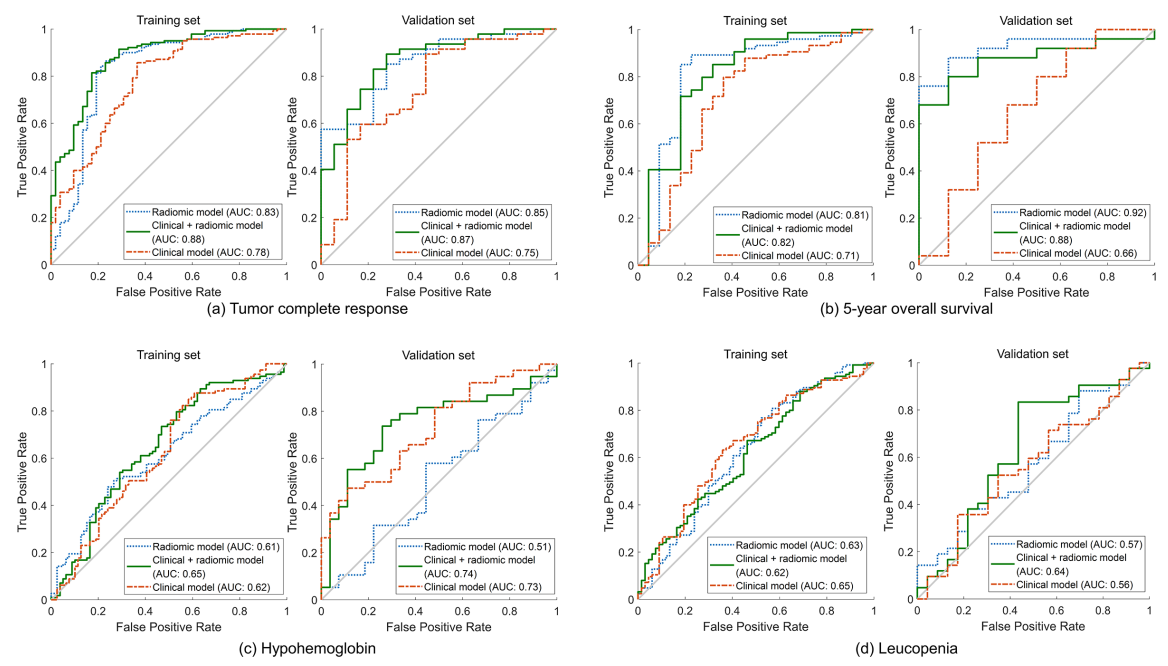


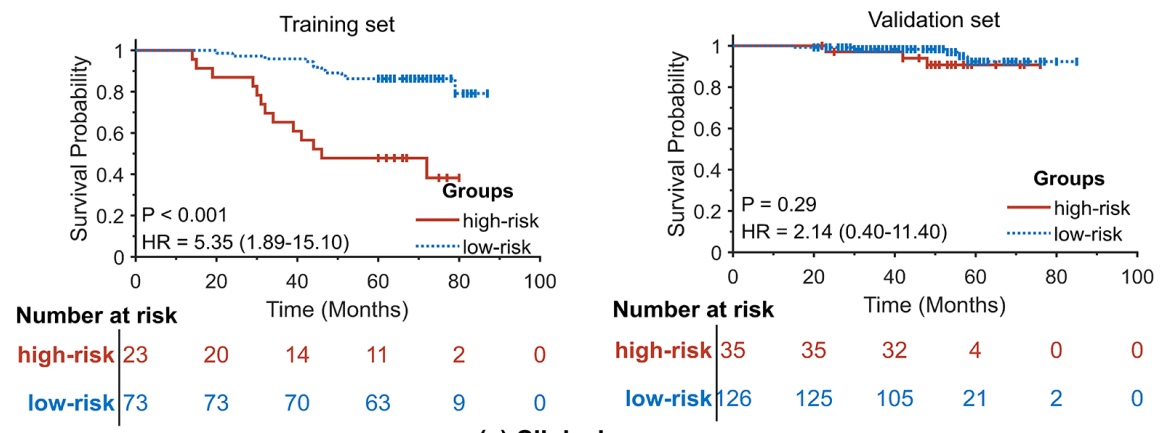

(a) Clinical score
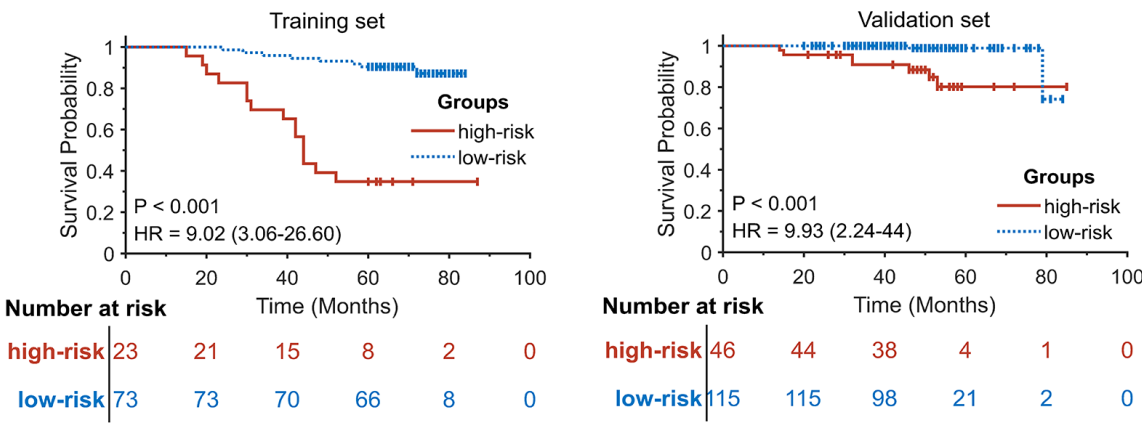

(b) Radiomic score
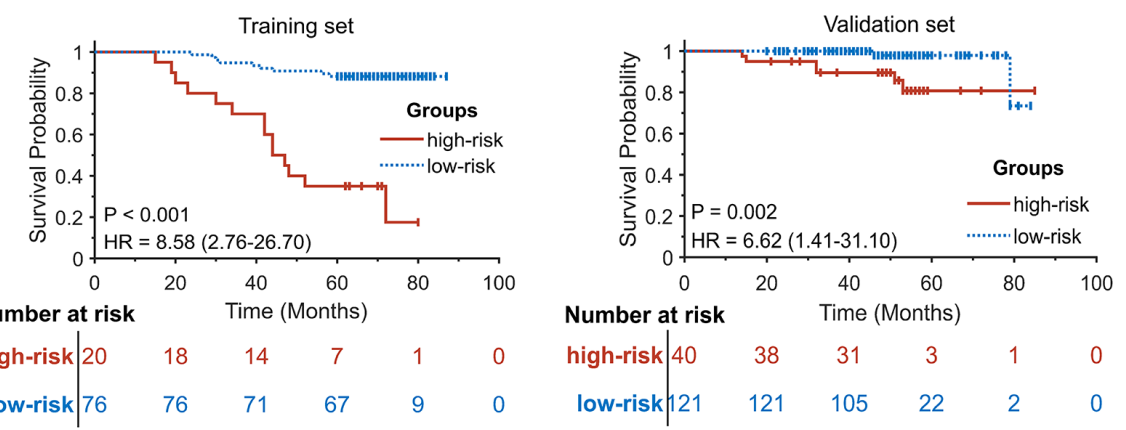

(c) Clinical and radiomic score 

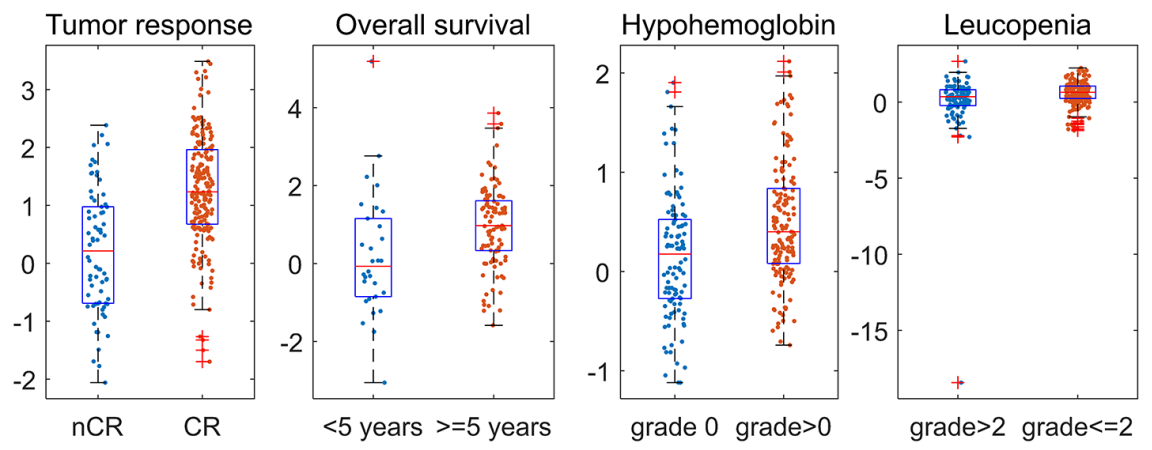

(a) Clinical score
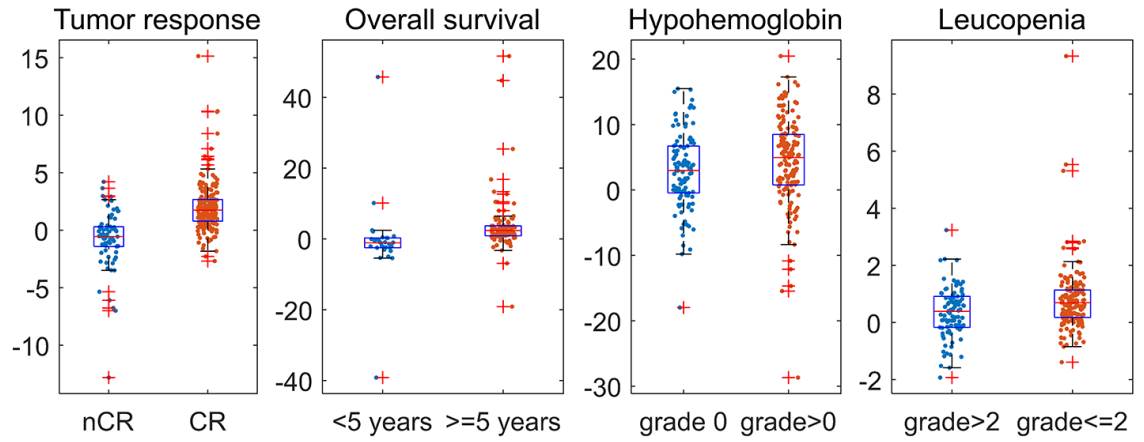

(b) Radiomic score
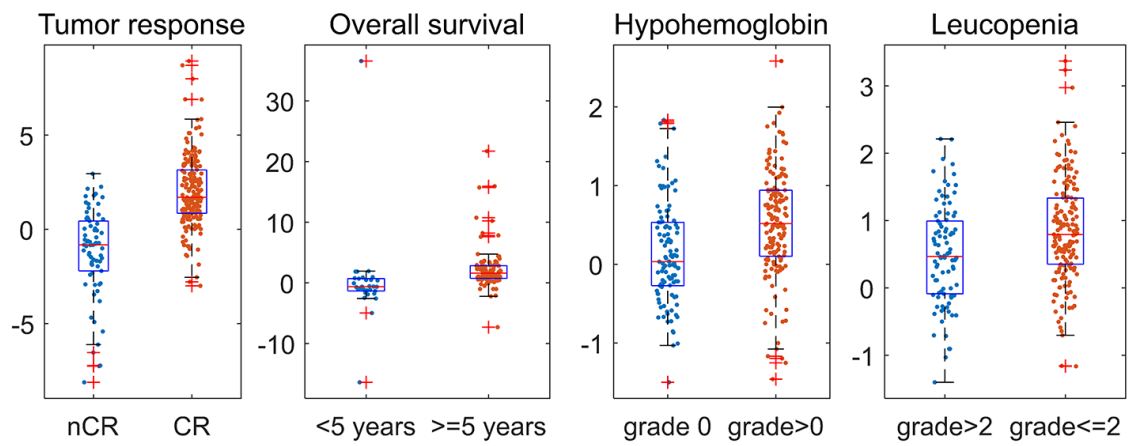

(c) Clinical and radiomic score 